SUPPORTING INFORMATION (SI)

\title{
DNA-Origami NanoTrap for Studying the Selective Barriers Formed by Phenylalanine-Glycine-Rich Nucleoporins
}

Qi Shen ${ }^{\mathrm{a}, \mathrm{b}, \mathrm{c}}$, Taoran Tian ${ }^{\mathrm{a}, \mathrm{b}}$, Qiancheng Xiong ${ }^{\mathrm{a}, \mathrm{b}}$, Patrick D. Ellis Fisher ${ }^{\mathrm{a}, \mathrm{b}}$, Yong Xiong ${ }^{\mathrm{c}}$, Thomas J. Melia ${ }^{\mathrm{a}}$, C. Patrick Lusk ${ }^{\mathrm{a}}$, and Chenxiang Lin ${ }^{\mathrm{a}, \mathrm{b}}$ *

${ }^{a}$ Department of Cell Biology, Yale University School of Medicine, 333 Cedar Street, New Haven, Connecticut 06520, United States

${ }^{b}$ Nanobiology Institute, Yale University, 850 West Campus Drive, West Haven, Connecticut 06516, United States

${ }^{c}$ Department of Molecular Biophysics and Biochemistry, 266 Whitney Avenue, Yale University, New Haven, Connecticut 06511, United States

*Email: patrick.lusk@yale.edu; chenxiang.lin@yale.edu 


\section{MATERIALS AND METHODS}

\section{General}

All chemicals were purchased from commercial sources and used without further purification unless otherwise stated. All DNA oligonucleotides were purchased from Integrated DNA Technologies (IDT).

\section{Cloning and Expression}

The coding sequences for amino acids 2-603 of Nsp1 and amino acids 2-610 of Nup100 from S. cerevisiae were cloned into 10×His-MBP-SUMO-nup-SNAP constructs (Figure 2A) via a pET-28a-derived vector (Novagen), and expressed in E. coli strain BL21-Gold (DE3). The coding sequences of GFP were cloned into the same vector, alone or with the MBP-SUMO-tag (Figure 3A) or S. cerevisiae Ntf2 (Figure 5) coding sequence. The plasmids were transformed into E. coli strain BL21-Gold (DE3) competent cells via heat shock. For expression, transformed bacteria were cultured in Luria Broth or Terrific Broth media with kanamycin $(50 \mu \mathrm{g} / \mathrm{mL})$ at $37^{\circ} \mathrm{C}$ while shaking at $220 \mathrm{rpm}$ for $3 \mathrm{hr}$, until $\mathrm{OD}_{600}$ reached $\sim 1.0$. IPTG $(1 \mathrm{mM})$ was then added to induce protein expression for $4-5 \mathrm{hr}$ at $25^{\circ} \mathrm{C}$ before cell collection by centrifugation. Cell pellets were stored at $-80^{\circ} \mathrm{C}$ until use.

\section{Protein purification}

The cell pellet was thawed and resuspended in lysis buffer ( $1 \times \mathrm{PBS}$ containing $150 \mathrm{mM} \mathrm{NaCl}, \mathrm{pH}$ 7.4, 0.1\% Tween 20, 0.1 mM PMSF, $1 \times$ Roche complete protease inhibitors), and lysed in a cell disruptor. Wholecell lysates were spun at 35k rpm for $45 \mathrm{~min}$ in a Type 45 Ti rotor (Beckman Coulter), and the supernatant was decanted and filtered through a $0.45 \mu \mathrm{m}$ cellulose acetate membrane. The resulting filtered lysate was applied to a $5 \mathrm{~mL}$ HisTrap column (GE Healthcare) on an ÄKTA FPLC system (GE Healthcare) at a 1 $\mathrm{mL} / \mathrm{min}$ flow rate. The column was washed with wash buffer ( $1 \times$ PBS, 0.1\% Tween 20, $25 \mathrm{mM}$ imidazole) and eluted on a gradient of elution buffer (1×PBS, 0.1\% Tween 20, 25-500 mM imidazole). Protein concentration was determined by Nanodrop (Thermo Fisher Scientific). Samples were flash-frozen in liquid nitrogen and stored at $-80^{\circ} \mathrm{C}$ until use.

\section{Benzylguanine (BG)-DNA preparation}

DNA anti-handles (5'-labeled amino-DNA oligonucleotides) were resuspended in deionized $\mathrm{H}_{2} \mathrm{O}$ at $2 \mathrm{mM}$. BG-GLA-NHS (New England BioLabs) was dissolved in DMSO at $20 \mathrm{mM}$. DNA anti-handles were then mixed with BG-GLA-NHS in a 1:3 volumetric ratio in $70 \mathrm{mM}$ HEPES buffer ( $\mathrm{pH} 8.5$ ) and incubated at room temperature (r.t.) for 1 hour. The BG-DNA product was then purified from excess BG-GLA-NHS by ethanol precipitation. Dried BG-DNA pellets were stored at $-20^{\circ} \mathrm{C}$ until use.

\section{Protein-DNA conjugation and purification}

BG-DNA pellets were resuspended in deionized $\mathrm{H}_{2} \mathrm{O}$ and mixed with purified nups in $1 \times$ PBS buffer to reach a final concentration of $40 \mu \mathrm{M}$ BG-DNA and $20 \mu \mathrm{M}$ SNAP-tagged protein (2:1 molar ratio). This reaction mixture was incubated at $25^{\circ} \mathrm{C}$ for 2 hours. Excess DNA was removed from conjugated proteins using size exclusion chromatography on a Superdex200 10/300 column (GE Healthcare) in 1×PBS buffer, pH 7.4, 0.1\% Tween 20. Conjugation efficiency was verified by SDS-PAGE (see below). 


\section{SDS-polyacrylamide gel electrophoresis (SDS-PAGE)}

All SDS-PAGE gels contained 8\% acrylamide bis-tris (Bio-Rad, pH 6.5). Samples were boiled in 1× Laemmli sample buffer at $90^{\circ} \mathrm{C}$ for 5 mins before loading to the gels. The gels were run for 40 min at 25 V/cm in MOPS-SDS buffer (50 mM Tris, $50 \mathrm{mM}$ MOPS, $1 \mathrm{mM}$ EDTA, 0.1\% SDS, pH 6.5). Gels were stained with Coomassie Blue or SYPRO Red (Thermo Fisher Scientific).

\section{DNA-origami design and assembly}

Channel and baseplate were designed in caDNAno ${ }^{1}$ (caDNAno.org), with bait and handle extending from the 3' end of staple strands at positions indicated in Figure 1D and S1. The extension sequences are 5'AAATTATCTACCACAACTCAC-3' (inner handle a), 5'-CTGATGATATTGATTGAAATG-3' (inner handle b), and 5'-CTTAAGCGATACGGGAATATG-3' (bait). The DNA-origami structures were assembled from an M13mp18 bacteriophage-derived circular ssDNA strand (8064 nt) and staple oligonucleotides (see Figure S1). The assembly was carried out using a $36 \mathrm{hr} 85^{\circ} \mathrm{C}-25^{\circ} \mathrm{C}$ annealing gradient in $1 \times$ TE buffer ( $5 \mathrm{mM}$ Tris-HCl, $1 \mathrm{mM}$ EDTA, pH 8.0) supplemented with $15 \mathrm{mM} \mathrm{MgCl}_{2}$ as reported previously. ${ }^{2}$ The assembled DNA channel and baseplate were then mixed at an equimolar ratio and incubated at $37^{\circ} \mathrm{C}$ for 48 hours for dimerization. The complete NanoTrap was purified using rate-zonal centrifugation $^{3}$ through a 15-45\% glycerol gradient in $1 \times \mathrm{TE}+10 \mathrm{mM} \mathrm{MgCl}_{2}$ in an SW 55 rotor (Beckman Coulter). Fractions were collected after a $1 \mathrm{hr}$ centrifugation at $50 \mathrm{k}$ rpm. Typically, $5 \mu \mathrm{L}$ of each fraction was loaded in a $1.5 \%$ agarose gel $\left(0.5 \times \mathrm{TBE}, 10 \mathrm{mM} \mathrm{MgCl}_{2}\right)$ with $0.5 \mu \mathrm{g} / \mathrm{mL}$ ethidium bromide (EtBr). A $1 \mathrm{~kb}$ DNA ladder (New England Biolabs) was run in parallel with samples. Electrophoresis was carried out at $5 \mathrm{~V} / \mathrm{cm}$ for $120 \mathrm{~min}$ in $0.5 \times \mathrm{TBE}, 10 \mathrm{mM} \mathrm{MgCl}_{2}$. Gels were imaged on a Typhoon FLA 9500 scanner (GE Healthcare). Fractions containing desired DNA structures (determined by agarose gel electrophoresis) were collected, and the buffer was changed to $1 \times \mathrm{TE}+10 \mathrm{mM} \mathrm{MgCl}_{2}$ using Amicon Ultra centrifugal filters with $100 \mathrm{kD}$ cutoff (EMD Millipore). The purified DNA nanostructures were then stored at $-20^{\circ} \mathrm{C}$.

\section{Attaching FG-nups to DNA NanoTrap}

DNA-conjugated nup was added to DNA NanoTraps at $\sim 2 \times$ excess over the number of handles (e.g., for 3 nM NanoTrap with 48 handles, $2 \times 3 \times 48 \approx 300 \mathrm{nM}$ FG-nup-DNA was added) in $1 \times$ TE buffer with $15 \mathrm{mM}$ $\mathrm{MgCl}_{2}$. The mixture was kept at $37^{\circ} \mathrm{C}$ for $2 \mathrm{hr}$ to allow handle-to-anti-handle hybridization. Optionally, the

products were purified by rate-zonal centrifugation, as described previously, ${ }^{2}$ through a $15-45 \%$ glycerol gradient in the hybridization buffer (1×TE buffer with $\left.15 \mathrm{mM} \mathrm{MgCl}_{2}\right)$.

\section{Permeability assay}

Sample preparation

We tested the diffusion barriers formed by FG-nups by incubating the FG-nup-gated NanoTraps with a series of fluorescently labeled molecules (reporters) that ranged from $7 \mathrm{kD}$ to $106 \mathrm{kD}$ : an Alexa488-prey (7 kD), a GFP-SNAP-prey (53 kD), and an MBP-GFP-SNAP-prey (106 kD). For testing NTR-mediated transport, a homodimer of Ntf2-GFP-SNAP-prey (140 kD) was used as the reporter to represent NTRbound cargo. Unless noted otherwise, $3 \mathrm{nM}$ NanoTraps containing various FG-nup configurations were incubated in separate test tubes with $1 \mu \mathrm{M}$ reporters of different sizes for 1 hours at $37^{\circ} \mathrm{C}$. Empty NanoTrap 
was incubated with the same set of reporters under identical conditions.

$\underline{\text { SDS-Agarose gel electrophoresis }}$

Samples were loaded in an SDS-agarose gel (1.5\% agarose in 0.5×TBE, $10 \mathrm{mM} \mathrm{MgCl}_{2}$, and $0.05 \%$ SDS). Electrophoresis was carried out at $5.8 \mathrm{~V} / \mathrm{cm}$ for $90 \mathrm{~min}$ in $0.5 \times \mathrm{TBE}$ buffer containing $10 \mathrm{mM} \mathrm{MgCl}_{2}$ and $0.05 \%$ SDS. Gels were imaged on a Typhoon FLA 9500 scanner (GE Healthcare) for the in-gel fluorescence (GFP or Alexa Fluor 488) first, stained with ethidium bromide (EtBr), and then imaged again for the $\mathrm{EtBr}$ fluorescence. For EtBr staining, the gel was first soaked in deionized $\mathrm{H}_{2} \mathrm{O}$ and shaken for $1 \mathrm{hr}$ to remove SDS, and then submerged in an EtBr solution (Sigma-Aldrich, 20,000× dilution in $\mathrm{H}_{2} \mathrm{O}$ to $0.5 \mu \mathrm{g} / \mathrm{mL}$ ) for $1 \mathrm{hr}$. Gels were destained for 1 hour in deionized $\mathrm{H}_{2} \mathrm{O}$ before imaging.

Image analysis

The gel images were analyzed using ImageJ (v2.1.0) using the built-in gel analyzing tool for the band intensities. To account for possible concentration variation among the NanoTrap samples, all NanoTrap bands' GFP/Alexa Fluor 488 fluorescence (from the trapped reporter molecules) were normalized against their $\mathrm{EtBr}$ fluorescence. The normalized fluorescence of the empty NanoTrap was set as a reference with 100\% penetration; the penetration of a certain reporter through an FG-nup-gated NanoTrap was quantified by dividing the normalized fluorescence of the NanoTrap band by that of the reference band and expressed as percentages (Figure 3-5).

\section{Negative-Stain Transmission Electron Microscopy}

Negative-stain TEM was used to visualize the DNA channel and baseplate, as well as empty and FG-nupgated NanoTraps. Typically, samples ( $5 \mu \mathrm{L}$ ) were loaded onto a glow discharged Formvar/carbon-coated copper grid (400 mesh, Electron Microscopy Sciences) and stained with 2\% uranyl formate. Imaging was performed on a JEOL JEM-1400 Plus microscope operated at $80 \mathrm{kV}$ with a bottom-mount $4 \mathrm{k} \times 3 \mathrm{k}$ CCD camera (Advanced Microscopy Technologies).

\section{Attaching AuNP to DNA NanoTrap}

Thiol-labeled prey-oligo (41 $\mu \mathrm{M})$ was mixed with phosphine-treated $5 \mathrm{~nm}$ AuNP (200 nM, Ted Pella) in $50 \mathrm{mM} \mathrm{NaCl}, 1 \times$ TBE buffer (44.5 mM Tris, $44.5 \mathrm{mM}$ boric acid, $1 \mathrm{mM}$ EDTA). ${ }^{4}$ The mixture was covered with aluminum foil and agitated in a ThermoMixer (Eppendorf) under r.t. at $300 \mathrm{rpm}$ for $\sim 40 \mathrm{hr}$. Subsequently, the DNA-conjugated AuNP was purified and washed with 0.5× TBE buffer using Amicon Ultra centrifugal filters with $50 \mathrm{kD}$ cutoff (EMD Millipore). To characterize the product, $5 \mu \mathrm{L}$ of AuNP was loaded in a 3\% agarose gel, which was run in $1 \times$ TAE buffer (40 mM Tris, $20 \mathrm{mM}$ acetic acid, $2 \mathrm{mM}$ EDTA) at $10 \mathrm{~V} / \mathrm{cm}$ for 30 mins (Figure S4). OD $_{520}$ of the resuspended AuNPs was measured to determine the AuNP concentration. The purified DNA-conjugated AuNPs were stored at $4{ }^{\circ} \mathrm{C}$ until use.

For AuNP attachment, the baseplate or empty NanoTrap (2 $\mathrm{nM}$ ) was incubated with prey-oligo-conjugated AuNP ( $2 \mathrm{nM}$ ) for 1.5 hours at $37^{\circ} \mathrm{C}$. The mixture was imaged by negative-stain EM to visualize the immobilization of AuNPs inside the NanoTraps.

\section{Statistical analysis}


The data analysis was performed using the SPSS 26.0 software package (IBM, United States). Unless noted otherwise, all statistical data were expressed in mean \pm standard error of the mean (SEM). Two-tailed ttests were applied to evaluate the differences between top and bottom arranged nucleoporins, two-way ANOVA and Tukey's multiple comparisons test was applied to evaluate the difference between mixed-nup NanoTraps and Nup100-only NanoTraps. Detailed statistics data were shown in Table S1. P $<0.05$ were considered statistically significant. 


\section{SUPPLEMENTARY FIGURES}

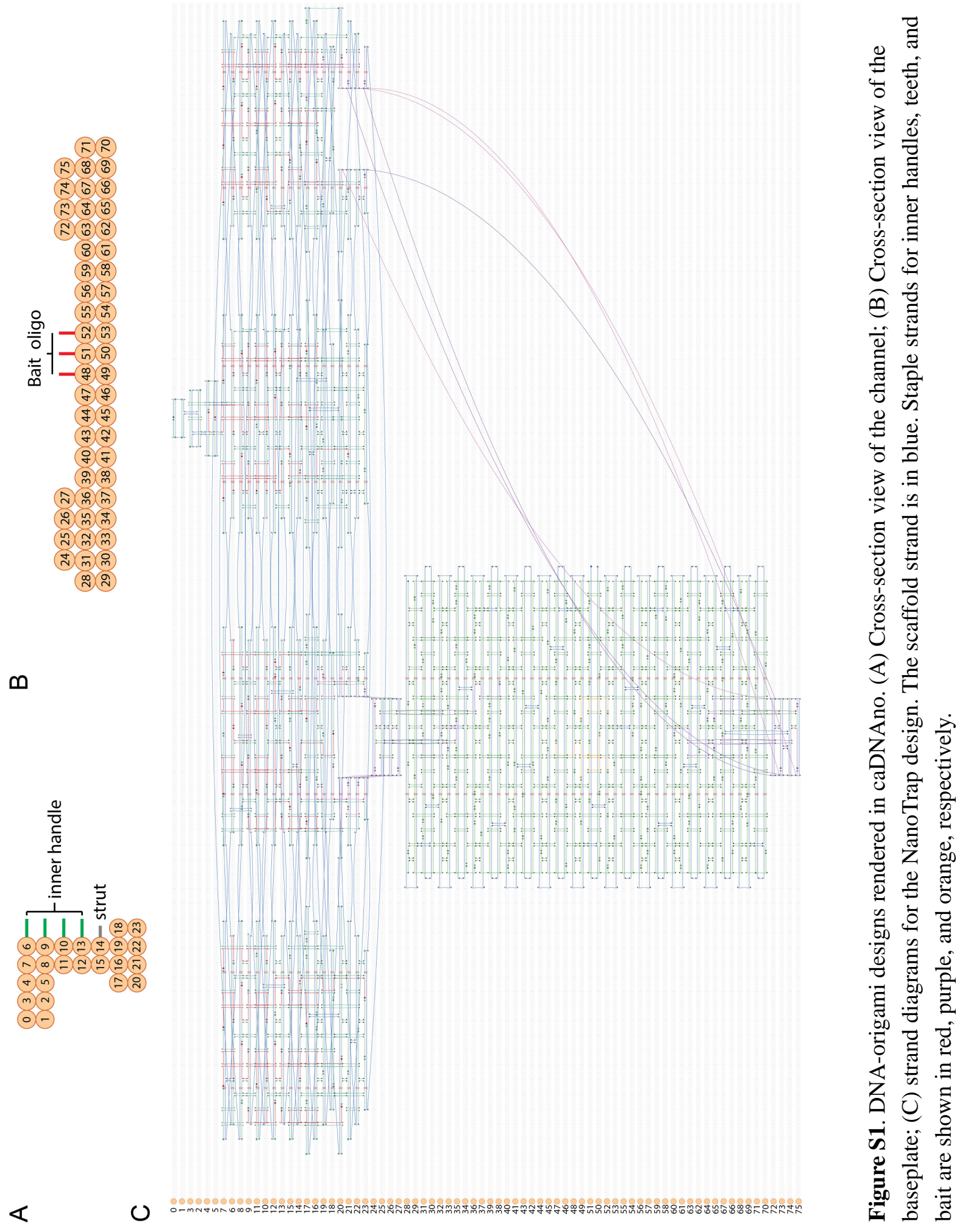




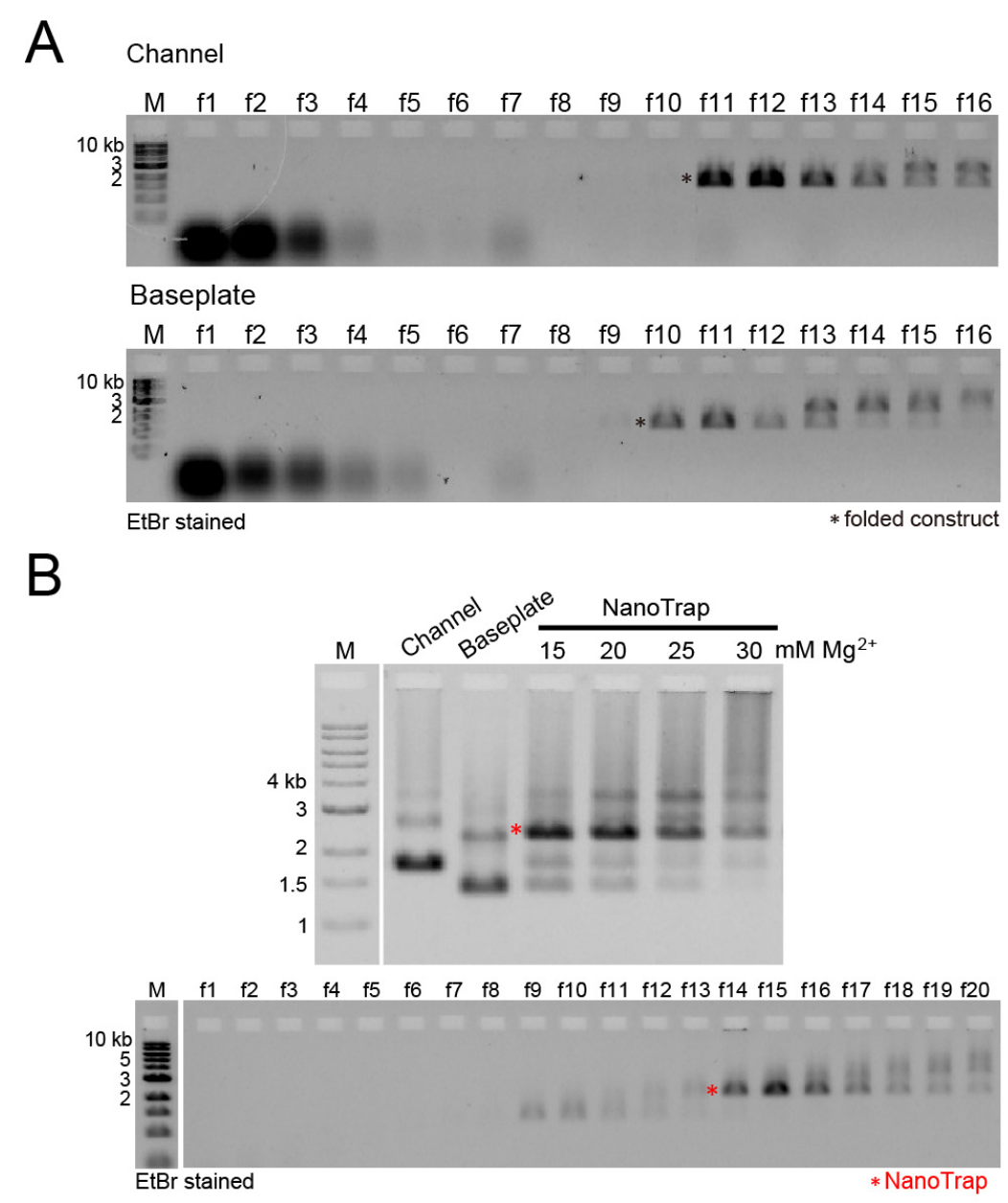

Figure S2. DNA-origami assembly and purification. (A) Folded DNA channel and baseplate purified by rate-zonal centrifugation. Agarose gel (1.5\%) electrophoreses show the enrichment of DNA channel (top) and baseplate (bottom) in fractions 11-13 and 10-12, respectively. Well-folded nanostructure bands are denoted by an asterisk; (B) Agarose gel electrophoreses show the channel and baseplate dimerization yield at different $\mathrm{MgCl}_{2}$ concentrations (top) and the enrichment of NanoTrap in fractions 14-16 after rate-zonal centrifugation (bottom). NanoTrap bands are denoted by an asterisk. 

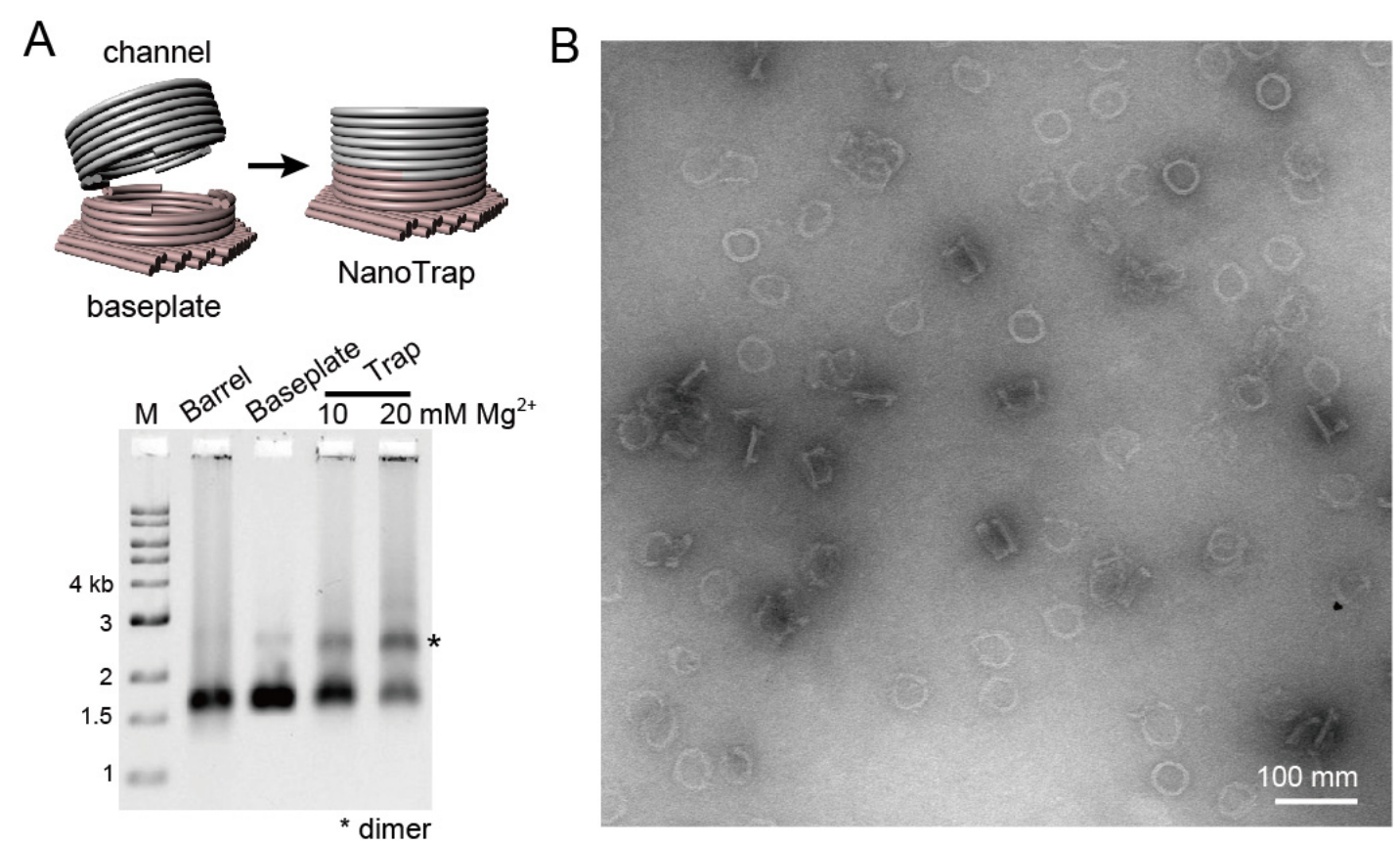

Figure S3. DNA NanoTrap with a ring-shaped channel. (A) Cartoon models of the ring-shaped NanoTrap assembly (top) and the analysis of dimerization yields by agarose gel electrophoresis (bottom); (B) Negative-stain TEM image of the ring-shaped NanoTrap. Scale bar: $100 \mathrm{~nm}$. Note the inferior assembly efficiency compared to the NanoTrap used in this study (Figure 1 and S2). 


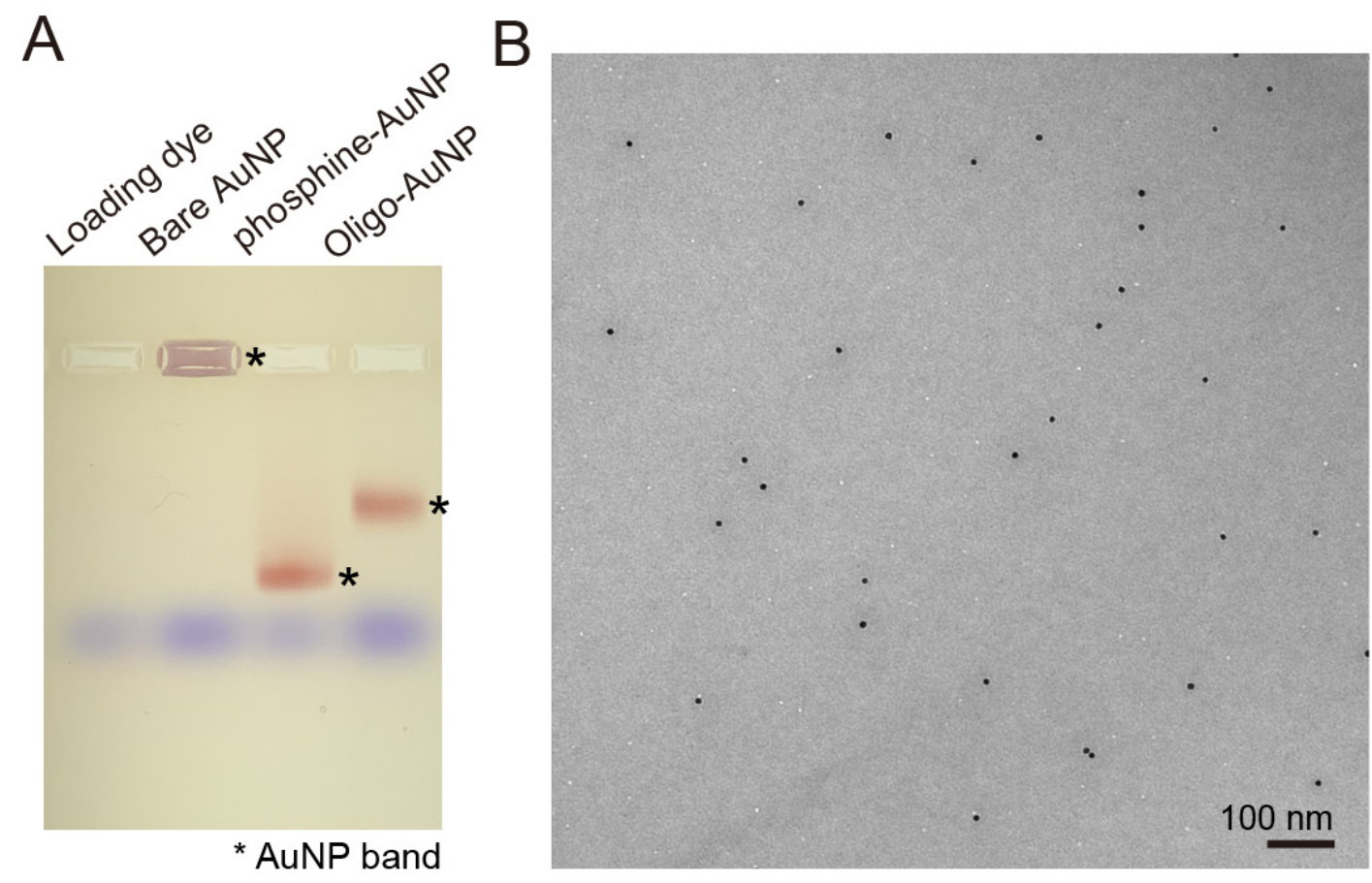

Figure S4. Preparation of prey-oligo-conjugated AuNP. (A) Agarose electrophoresis showing the different mobilities of bare AuNP (stuck in the well), phosphine-treated AuNP, and prey-oligo-conjugated AuNP. AuNP bands are denoted by an asterisk. (B) A TEM image of the prey-oligo-conjugated $5 \mathrm{~nm}$ AuNPs with no signs of aggregation. Scale bar: $100 \mathrm{~nm}$. 

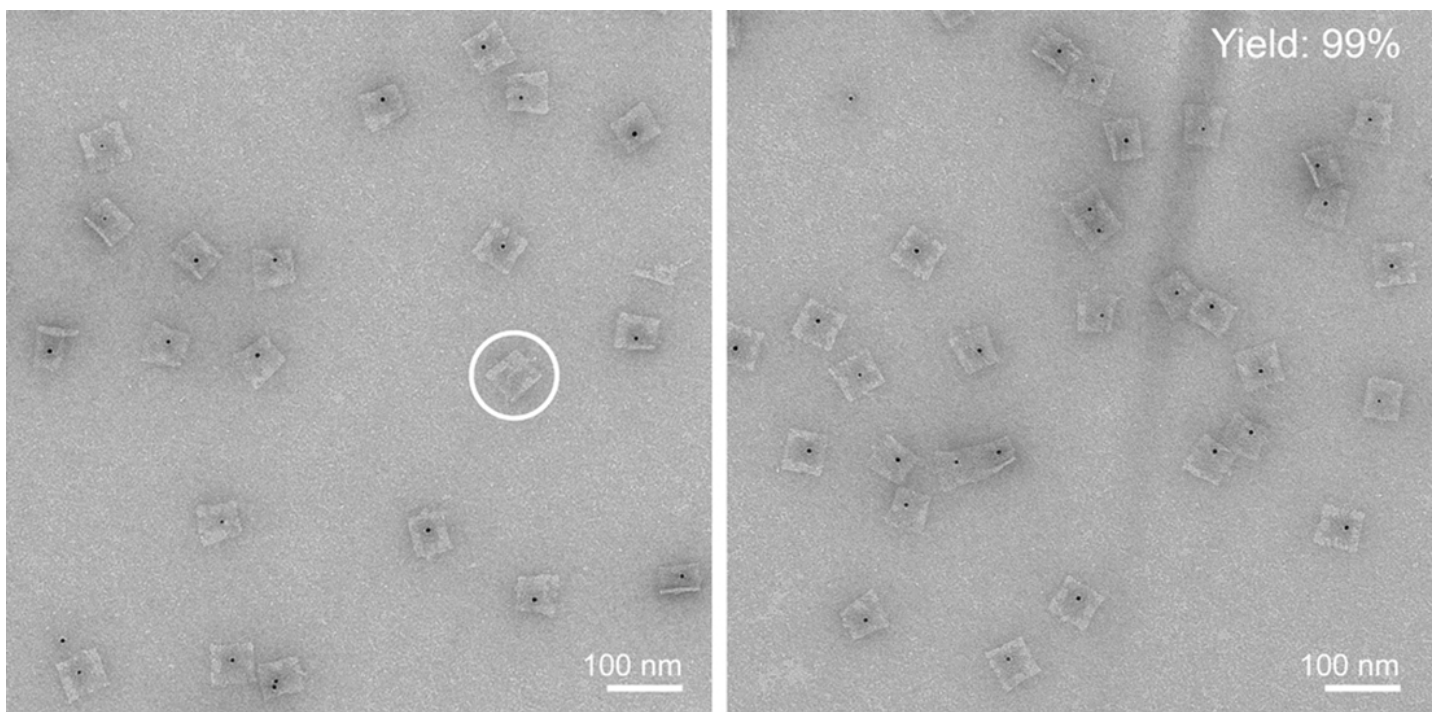

Figure S5. DNA-origami baseplates capture AuNPs via prey-bait hybridization. Negative-stain EM images show that the prey-oligo-conjugated $5 \mathrm{~nm}$ AuNPs (dark spots) are immobilized on the prey-oligo-displaying baseplates with a $\sim 99 \%$ yield $(\mathrm{N}=173)$. A baseplate without AuNP labeling is marked by a white circle. Scale bars: $100 \mathrm{~nm}$. 


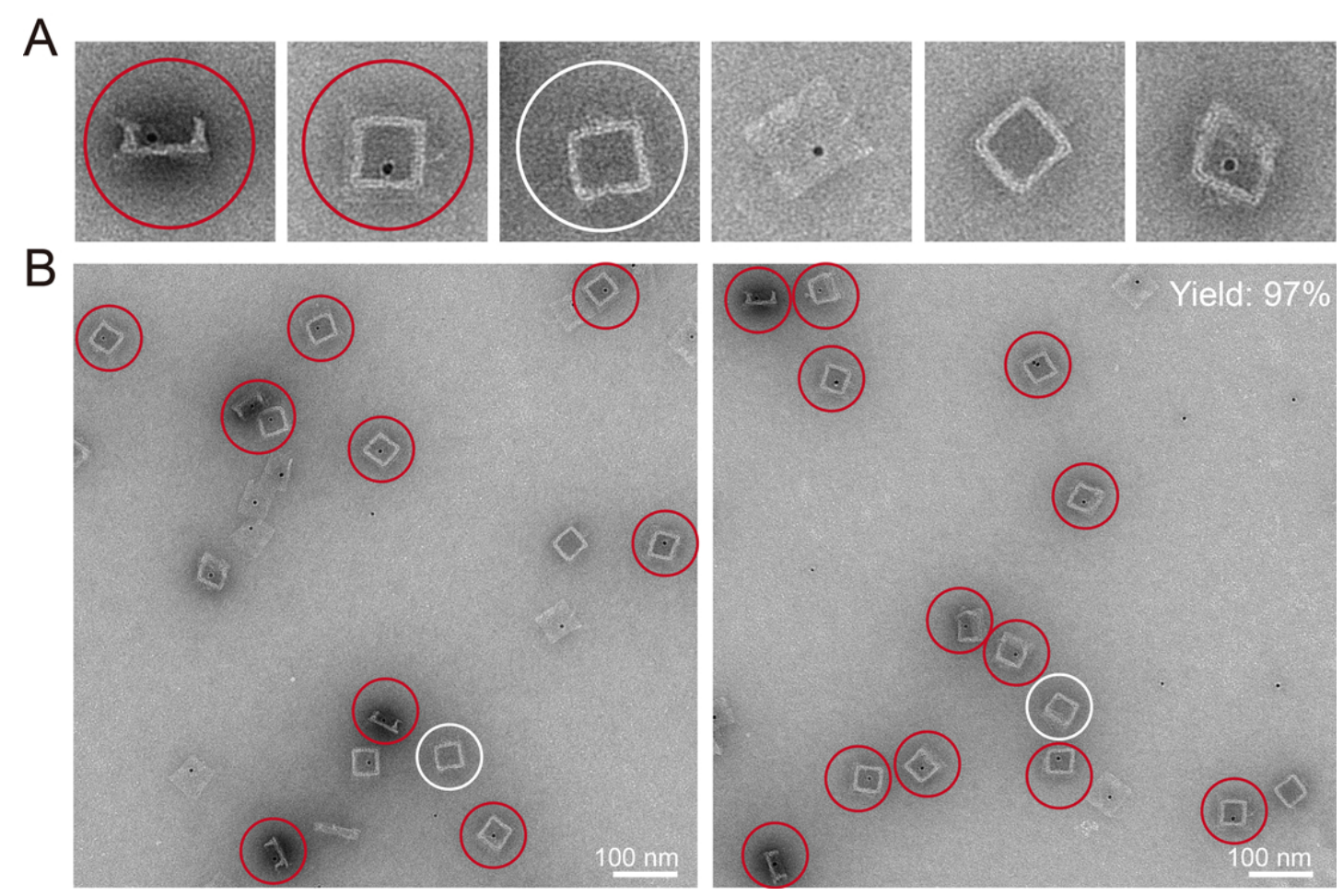

Figure S6. Empty NanoTraps capture AuNPs via prey-bait hybridization. (A) Zoom-in EM images showing representative views of different DNA-origami objects, from left to right: AuNP-labeled NanoTraps (marked by red circles), a AuNP-free NanoTrap (marked by a white circle), incomplete NanoTraps (baseplate or channel only), and an ambiguous case. (B) Representative zoom-out EM images showing that the prey-oligo-conjugated $5 \mathrm{~nm}$ AuNPs (dark spots) are immobilized on the prey-oligo-displaying baseplates of the NanoTraps with a $\sim 97 \%$ yield ( $N=129)$. Yield = (number of AuNP-labeled NanoTraps) / (total number of correctly assembled NanoTraps). Scale bars: $100 \mathrm{~nm}$. 

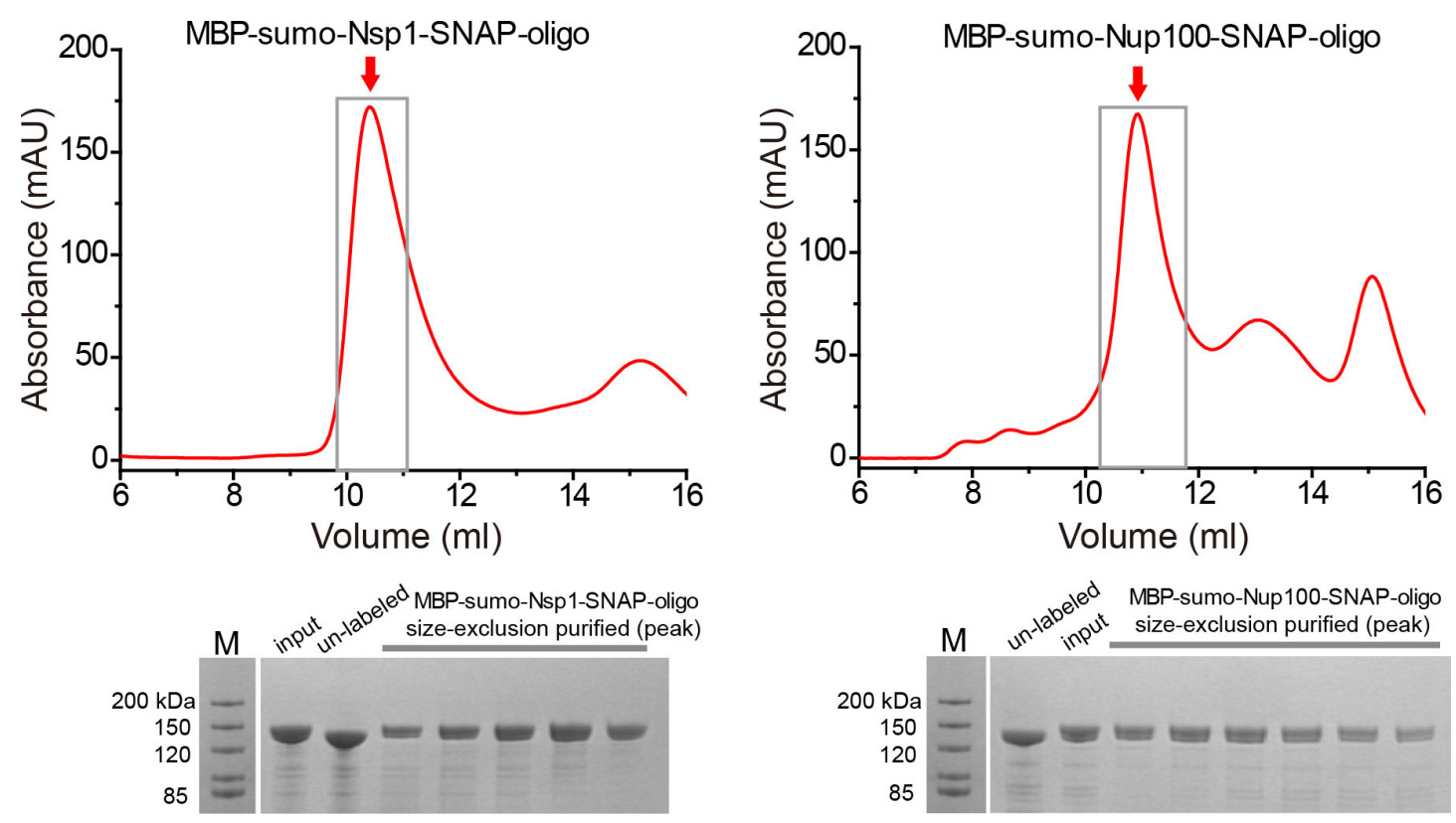

Figure S7. MBP-sumo-Nsp1-SNAP-oligo and MBP-sumo-Nup100-SNAP-oligo purification. The DNAconjugated Nsp1 and Nup100 are marked by a red arrow in their respective size exclusion chromatography graphs. SDS-PAGE show the fractions containing purified nup-DNA conjugates (denoted by a gray box in the chromatography graphs). 


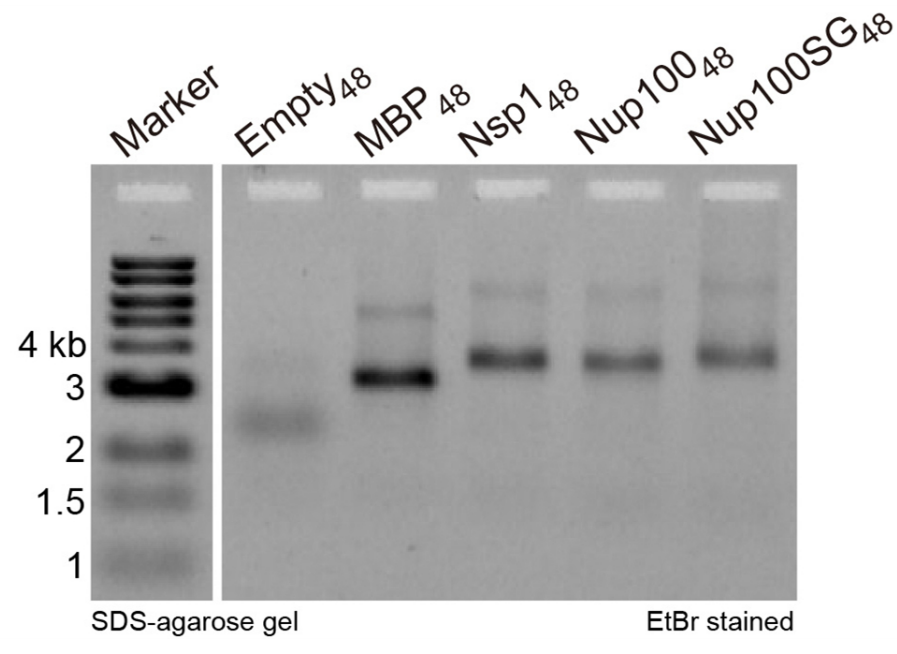

Figure S8. NanoTraps characterized by SDS-agarose gel electrophoresis. 

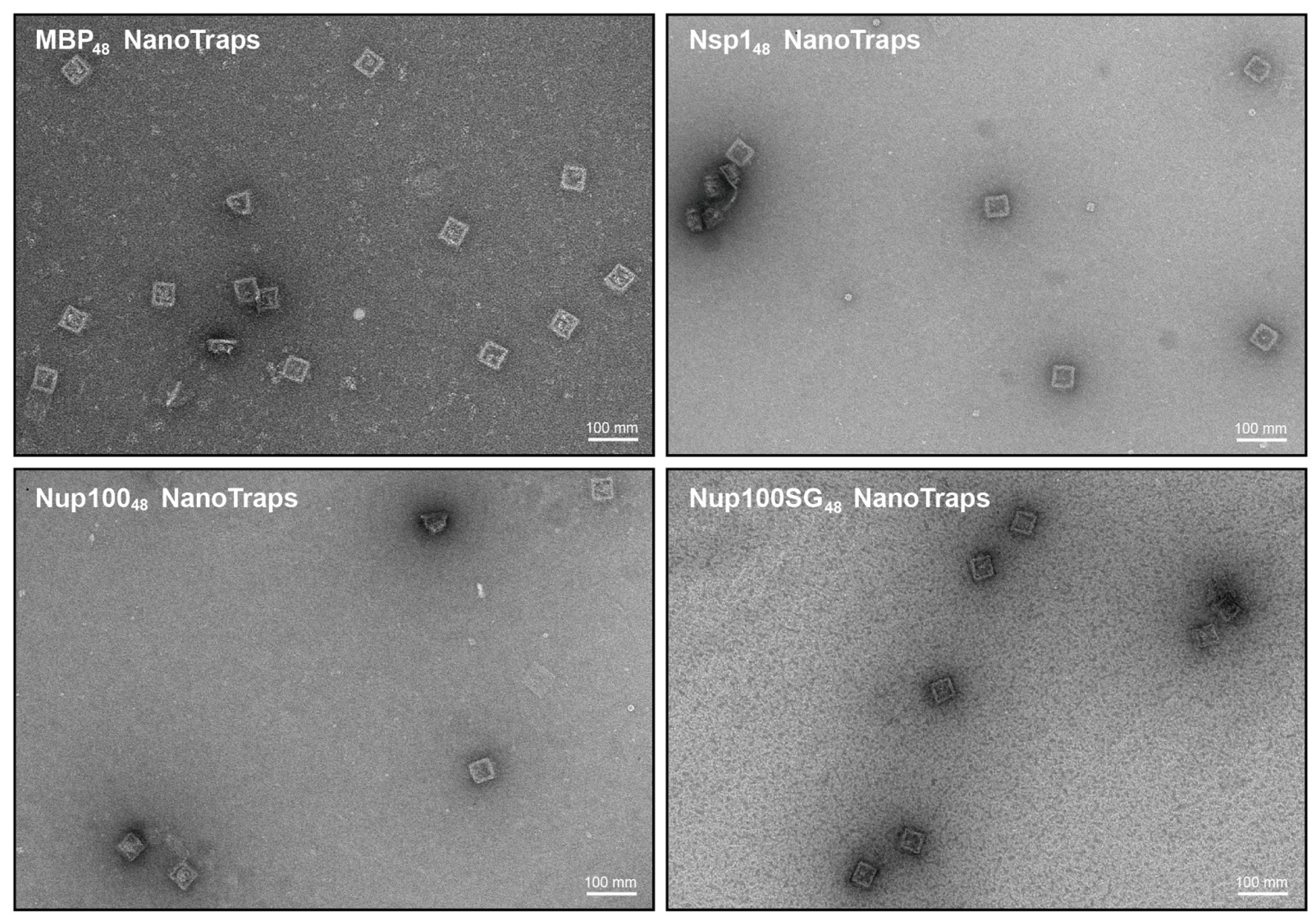

Figure S9. Negative-stain EM images of various protein-gated NanoTraps. 

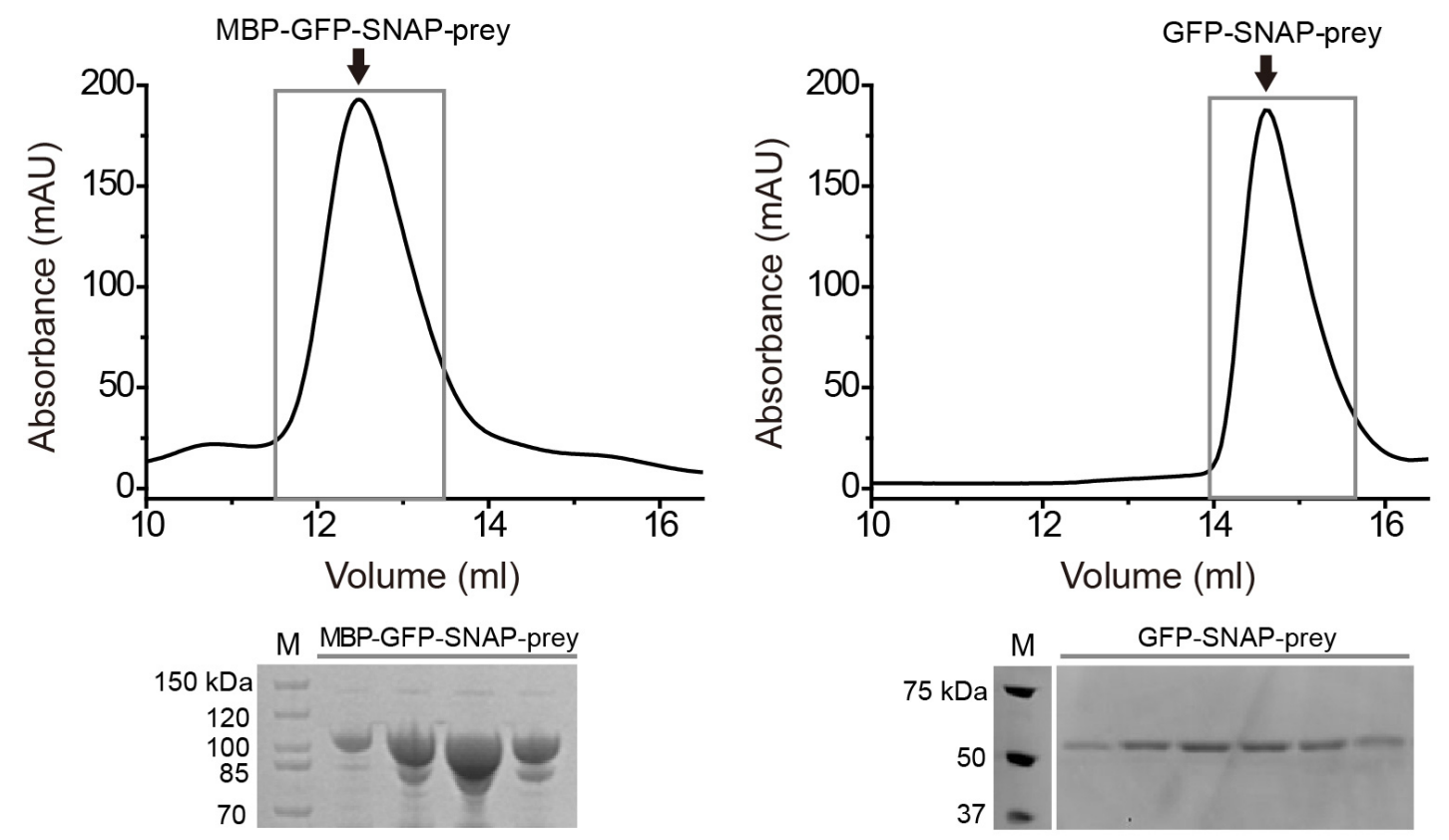

Figure S10. MBP-GFP-SNAP-prey and GFP-SNAP-prey purification. The DNA-conjugated MBP-GFPSNAP and GFP-SNAP are marked by an arrow in their respective size exclusion chromatography graphs. SDS-PAGE show the fractions containing purified protein-DNA conjugates (denoted by a gray box in the chromatography graphs). 


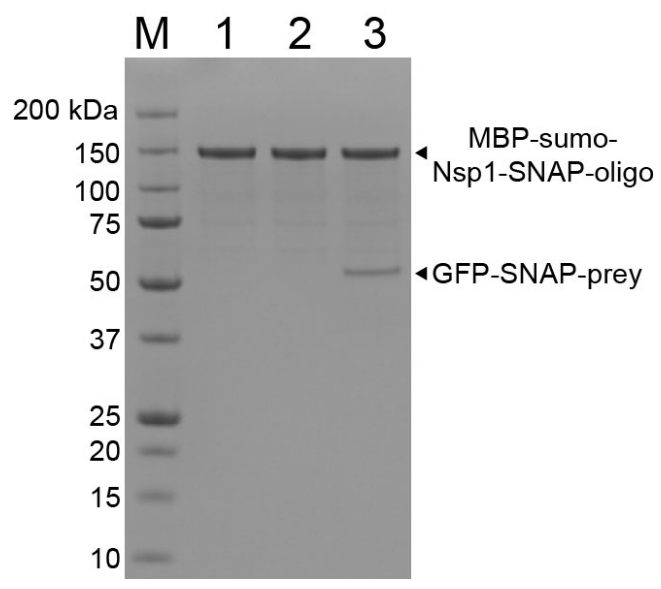

Figure S11. SDS-PAGE on Nsp1-antihandle conjugate alone (lane 1), after incubation with NanoTraps at $37^{\circ} \mathrm{C}$ for $2 \mathrm{hr}$ (lane 2), and after incubation with NanoTraps and GFP-SNAP-prey for $3 \mathrm{hr}$ (lane 3). No sign of degradation was detected. 

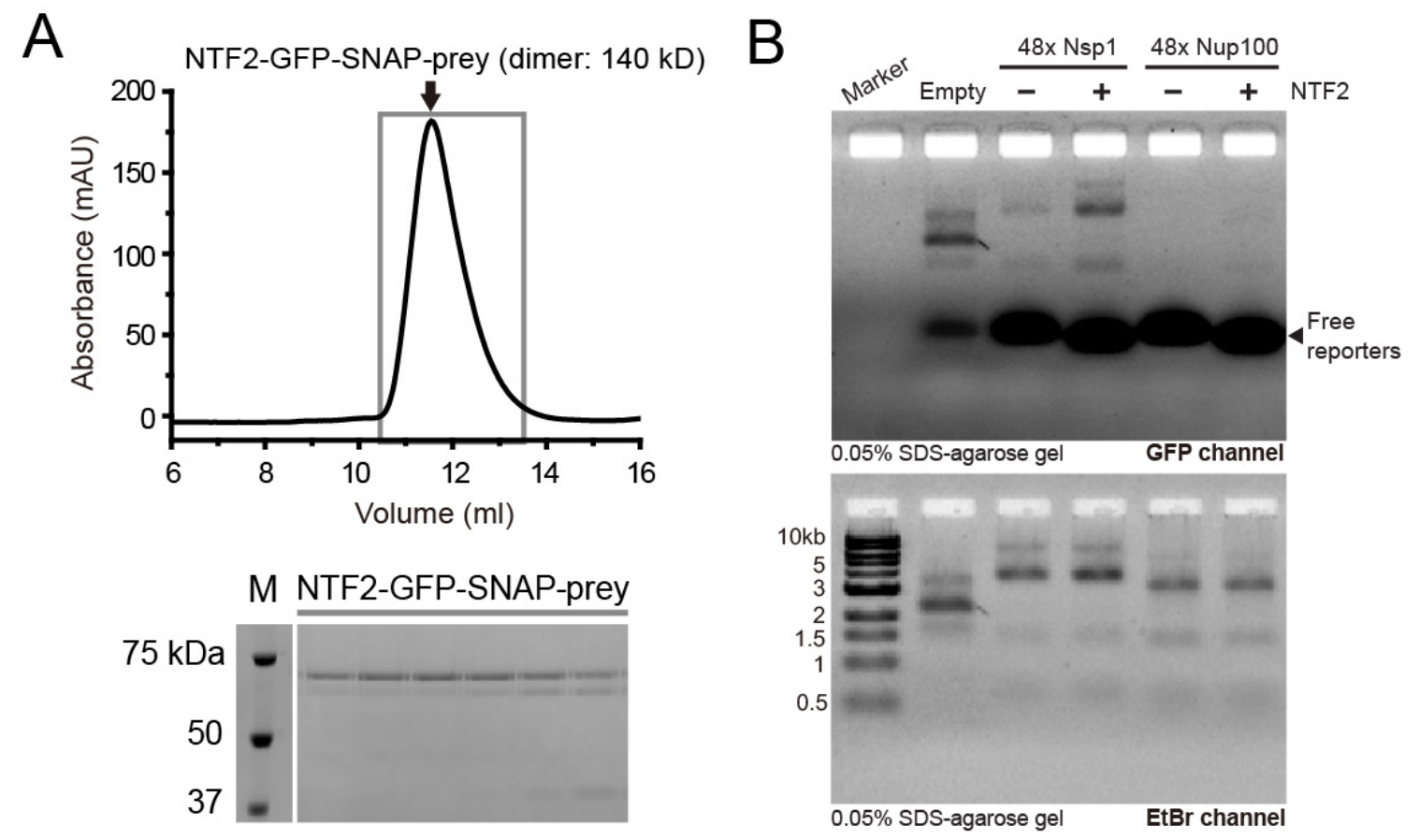

Figure S12. NTR-mediated cargo transport through FG-nup gated NanoTraps. (A) Ntf2-GFP-SNAP-prey purification. The DNA-conjugated Ntf2-GFP-SNAP is marked by an arrow in the size exclusion chromatography graph (top). The elusion volume of the protein-DNA conjugate indicates a monodispersed dimer population. SDS-PAGE (bottom) resolves the purified protein-DNA conjugate (fractions denoted by a gray box in the chromatography graphs) in its monomeric form. (B) Full-length agarose gel images showing the permeability of Nsp1 48 and Nup100 ${ }_{48}$-NanoTraps to Ntf2-GFP-SNAP-prey dimer (+Ntf2 lanes) and MBP-GFP-SNAP-prey (-Ntf2 lanes). 
Table S1. Data of statistical analysis

\begin{tabular}{|c|c|c|c|c|c|c|}
\hline \multicolumn{7}{|c|}{ Statistics data of t-test (Nup100 located at top and bottom of the NanoTrap) } \\
\hline & F-test (F, DFn, Dfd) & P-value of F-test & \multicolumn{2}{|c|}{ t-test (t, df) } & \multicolumn{2}{|c|}{ P-value } \\
\hline 12 top vs 12 bottom & 2.337, 2, 2 & 0.5993 & \multicolumn{2}{|c|}{$7.821,4$} & \multicolumn{2}{|c|}{0.0014} \\
\hline 24 top vs 24 bottom & $2.252,2,2$ & 0.615 & \multicolumn{2}{|c|}{ 2.711, 4} & \multicolumn{2}{|c|}{0.0535} \\
\hline 36 top vs 36 bottom & $1.286,2,2$ & 0.875 & \multicolumn{2}{|c|}{$1.744,4$} & \multicolumn{2}{|c|}{0.1562} \\
\hline \multicolumn{7}{|c|}{ Statistics data of t-test (Nsp1 located at top and bottom of the NanoTrap) } \\
\hline & F-test (F, DFn, Dfd) & P-value of F-test & \multicolumn{2}{|c|}{ t-test (t, df) } & \multicolumn{2}{|c|}{ P-value } \\
\hline 12 top vs 12 bottom & $3.910,2,2$ & 0.4073 & \multicolumn{2}{|c|}{ 0.5298, 4} & \multicolumn{2}{|c|}{0.6243} \\
\hline 24 top vs 24 bottom & $1.912,2,2$ & 0.6869 & \multicolumn{2}{|c|}{$0.2244,4$} & \multicolumn{2}{|c|}{0.8335} \\
\hline 36 top vs 36 bottom & $1.981,2,2$ & 0.6709 & \multicolumn{2}{|c|}{$2.424,4$} & \multicolumn{2}{|c|}{0.0725} \\
\hline \multicolumn{7}{|c|}{ Statistics data of Tukey's multiple comparisons test (mixed vs Nup100) } \\
\hline Test details & Mean 1, mean2, mean diff. & $95.00 \% \mathrm{CI}$ & f diff. & $\mathrm{N} 1, \mathrm{~N} 2$ & Q-value, df & P-value \\
\hline Empty ${ }_{36}$ Nup $100_{12}$ & 15.63, 33.98, -18.35 & -35.24 to & .91 & 3,3 & $3.755,36$ & 0.0308 \\
\hline Nup100 12 Empty $_{36}$ & $40.62,71.39,-30.77$ & -47.66 to & & 3,3 & $6.296,36$ & 0.0002 \\
\hline Empty $24 \mathrm{Nup} 100_{24}$ & $15.34,17.16,-1.821$ & -18.71 to & & 3,3 & $0.3727,36$ & 0.9625 \\
\hline Nup10024Empty24 & $29.41,25.14,4.265$ & -12.63 to & & 3,3 & $0.8728,36$ & 0.8117 \\
\hline Empty ${ }_{12}$ Nup $100_{36}$ & $19.8,14.6,5.2$ & -11.69 to & & 3,3 & $1.064,36$ & 0.7341 \\
\hline Nup10036Empty12 & $24.23,22.37,1.853$ & -15.04 to & & 3,3 & $0.3791,36$ & 0.9612 \\
\hline
\end{tabular}

\section{REFERENCES}

1. Douglas, S. M.; Marblestone, A. H.; Teerapittayanon, S.; Vazquez, A.; Church, G. M.; Shih, W. M., Rapid prototyping of 3D DNA-origami shapes with caDNAno. Nucleic Acids Res 2009, 37 (15), 5001-5006.

2. Fisher, P. D. E.; Shen, Q.; Akpinar, B.; Davis, L. K.; Chun, K. K. H.; Baddeley, D.; Saric, A.; Melia, T. J.; Hoogenboom, B. W.; Lin, C. X.; Lusk, C. P., A Programmable DNA Origami Platform for Organizing Intrinsically Disordered Nucleoporins within Nanopore Confinement. Acs Nano 2018, 12 (2), 1508-1518.

3. Lin, C. X.; Perrault, S. D.; Kwak, M.; Graf, F.; Shih, W. M., Purification of DNA-origami nanostructures by rate-zonal centrifugation. Nucleic Acids Res 2013, 41 (2), e40.

4. Shen, X. B.; Song, C.; Wang, J. Y.; Shi, D. W.; Wang, Z. A.; Liu, N.; Ding, B. Q., Rolling Up Gold Nanoparticle-Dressed DNA Origami into Three-Dimensional Plasmonic Chiral Nanostructures. J Am Chem Soc 2012, 134 (1), 146-149. 